\title{
Targeting heat shock transcription factor 1 for novel hyperthermia therapy (Review)
}

\author{
YOSHIAKI TABUCHI $^{1}$ and TAKASHI KONDO ${ }^{2}$ \\ ${ }^{1}$ Division of Molecular Genetics Research, Life Science Research Center, ${ }^{2}$ Department of Radiological Sciences, \\ Graduate School of Medicine and Pharmaceutical Sciences, University of Toyama, Toyama 930-0194, Japan
}

Received December 21, 2012; Accepted March 14, 2013

DOI: $10.3892 /$ ijmm.2013.1367

\begin{abstract}
Hyperthermia (HT) has shown promising antitumor effects against various types of malignant tumors, and its pleiotropic effects support its combined use with radiotherapy and/or chemotherapy. However, HT is rendered less effective by the acquisition of thermoresistance in tumors, which arises through the elevation of heat shock proteins (HSPs) or other tumor responses. In mammals, the induction of HSPs is principally regulated at the transcriptional level by the activation of heat shock transcription factor 1 (HSF1). This transactivator has been shown to be abundantly expressed in a wide variety of tumors in humans. In addition, HSF1 participates in the initiation, proliferation and maintenance of tumors. Of note, HSF1 silencing has been shown to prevent the progression of tumors and to enhance their sensitivity to HT. Here, we review the physiological and pathological roles of HSF1 in cancer cells, and discuss its potential as a therapeutic target for HT therapy.
\end{abstract}

\section{Contents}

1. Introduction

2. Function of HSF1

3. HSF1 and cancer

4. HSF1 and hyperthermia

5. Discussion

Correspondence to: Dr Yoshiaki Tabuchi, Division of Molecular Genetics Research, Life Science Research Center, University of Toyama, 2630 Sugitani, Toyama 930-0194, Japan

E-mail: ytabu@cts.u-toyama.ac.jp

Abbreviations: BMP, bone marrow progenitor; DN, dominantnegative; ER, estrogen receptor; HER2, epidermal growth factor receptor-2; HSE, heat shock element; HSP, heat shock protein; HSF, heat shock transcription factor; HSF1, heat shock transcription factor 1; HT, hyperthermia; KO, knockout; MTA1, metastasis-associated 1; MEF, mouse embryonic fibroblast; OSCC, oral squamous cell carcinoma; $\mathrm{p}-\mathrm{TEFb}$, positive transcription elongation factor $\mathrm{b}$; shRNA, short hairpin RNA; siRNA, small interfering RNA

Key words: hyperthermia, heat shock transcription factor 1, cancer

\section{Introduction}

Hyperthermia (HT) is considered to have potential as a cancer treatment modality (1). HT in combination with radiotherapy and/or chemotherapy has been used for various types of cancer, and the anticancer effects of such combinations have been verified in many clinical trials (1-7). One of the problems with HT therapy is the acquisition of thermoresistance against heat stress (8-12). In general, cells have protective functions for various stressors that occur from outside of the cell. Heat shock proteins (HSPs), molecular chaperones with strong cytoprotective and antiapoptotic properties, are induced by a wide variety of stresses, particularly heat stress (13-16); they are also induced by treatment with HT, and, thus, it has been considered that HSPs play a role in the acquisition of thermoresistance in cells (10-12). In mammals, the expression of HSPs is mainly regulated by heat shock transcription factor 1 (HSF1) $(17,18)$. Elevation of HSF1 has been observed in several types of human tumor tissues (19-26), and it has been shown to participate in the initiation, proliferation and maintenance of tumors $(12,25-29)$. Notably, both inhibition of the expression of HSF1 and the functional loss of HSF1 have been suggested to enhance the sensitivity to HT under basic experimental conditions (29-38). In the present review, the physiological and pathological roles of HSF1 in cancer cells are summarized, and the potential of HSF1 as a therapeutic target for HT therapy is discussed.

\section{Function of HSF1}

The heat-shock response, which is a universal cellular response to heat, is a critical cellular event for cell adaptation. Heat stress elicits a wide spectrum of stress responses, including an induction of HSPs, protein aggregation, an imbalance of protein homeostasis, DNA and RNA damage, reactive oxygen species production, cell growth arrest and cell death in mammalian cells. HSPs are characterized as molecular chaperones and they exert strong cytoprotective effects against stress-induced proteotoxic damage (13-16). Their functions and amino acid sequences are well conserved in a wide range of species from yeast to humans. The expression of HSPs is primarily regulated by heat shock transcription factors (HSFs). In humans, three HSFs (HSF1, HSF2 and HSF4) have been identified and, among them, HSF1 plays the most important 
role against stress responses $(17,18)$. Under nonstress conditions, HSF1 is localized in the cytoplasm as an inactive monomer. Upon exposure to stresses, especially heat stress, a series of events is triggered: the HSF1 inactive monomer is converted to a DNA-binding homotrimer that translocates from the cytoplasm to the nucleus, binds to the heat shock elements (HSEs) that are located in the regions of inducible HSP genes including Hsp27, Hsp70 and Hsp90, undergoes a hyperphosphorylation reaction and activates transcription $(17,18,39)$ (Fig. 1). In mammalian cells, heat activates the transcription of heat responsive genes, including HSPs, coincident with a bulk decrease in mRNA and protein syntheses, and this overall reprogramming of gene expression permits the selective synthesis of HSPs (40-42). In our previous studies using global-scale microarrays, we also detected a number of genes that were downregulated and participated in biological functions, including post-transcriptional modification and gene expression (43-45). This bulk decrease has been considered to represent a form of transcriptional suppression due to the binding of activated HSF1 to the promoter region of the gene (40). Mariner et al (41) recently reported that non-corded RNA is very closely related to the mechanism by which heat induces a decrease in gene expression.

Numerous studies targeting HSFs in gene-modified animals have enhanced our understanding of the pleiotropic roles of HSFs in mammals (46). A previous study with HSF1knockout (KO) mice demonstrated that the HSF1 protein was dispensable for the maintenance of the mice, since the KO mice remained alive under normal environmental conditions (30). On the other hand, HSF1 is reported to be required for extraembryonic development, postnatal growth and fertility of the female in the HSF1-KO mice (47). Homma et al (48) observed hallmarks of central nervous system disease, such as demyelination, astrogliosis and accumulation of ubiquitinated proteins in HSF1-deficient mice. Furthermore, previous studies with HSF1 KO animals have shown that HSF1 is involved in the development and maintenance of several organs, such as the heart (49), trachea (50), nose (50) and placenta (47). It has also been demonstrated that both HSF2 (51) and HSF4 (52) play critical roles in the neural functions or lens development by using HSF2- and HSF4-KO mice. Thus, accumulating evidence suggests that HSFs are closely associated with longevity (53), neurodegenerative diseases (54) and cancer (12,25-29). Indeed, HSFs play a versatile role in the organism, from homeostatic maintenance to disease.

\section{HSF1 and cancer}

In humans, tumorigenesis is a multistep development including genome instabilities (mutation and chromosomal deletion) and epigenetic changes (abnormal histone acetylation and DNA methylation). During the multistep tumorigenesis, mutated nonnative proteins are synthesized and deregulated and abnormal signal transduction pathways exist in cancer cells. Moreover, the conditions within the tumor microenvironment where cancer cells are present include hypoxia, acidity, and low glucose levels, and these differ from the conditions in normal tissue. Therefore, cancer cells are generally exposed to more stresses compared with normal cells (55). Several types of tumors contain high expression levels of one or more HSPs

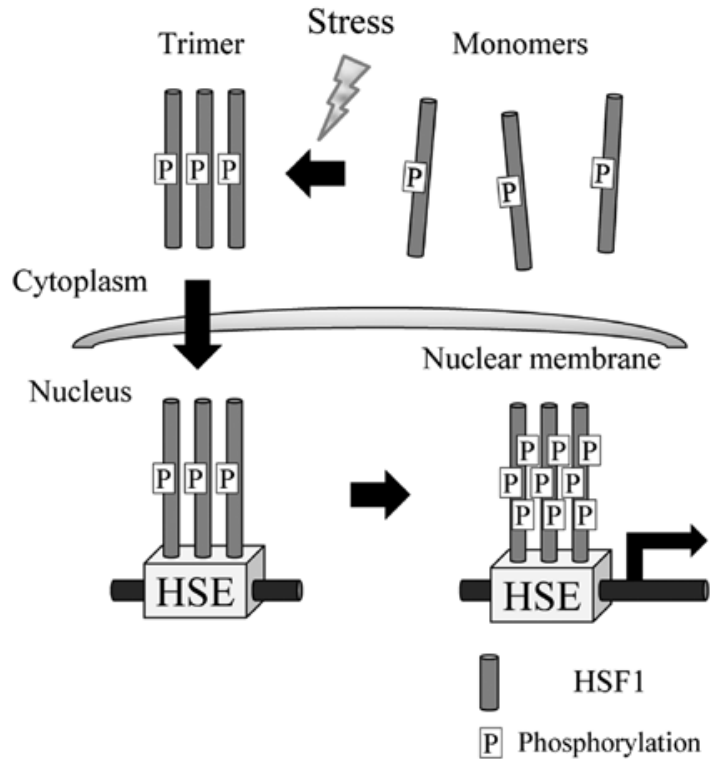

Figure 1. Schematic illustration of the activation of HSF1. HSF1 exists as an inactive monomer in the cytoplasm under nonstress conditions. Upon the introduction of stresses, especially heat stress, the HSF1 monomer is converted to a DNA-binding homotrimer that translocates from the cytoplasm to the nucleus. Then, the homotrimer binds to the HSE in the promoter of the gene, undergoes a hyperphosphorylation reaction and activates transcription.

(Hsp27,Hsp40,Hsp60,Hsp70, Hsp90 and/or Hsp110) compared with adjacent normal tissues $(12,56,57)$. A significant elevation of HSF1 has been reported in a wide variety of cancer cells or tumor tissues in nonclinical studies (19-26) (Table I). In human prostate carcinoma cell lines, the expression levels of HSPs and HSF1 are elevated in cells with more highly malignant features $(19,20)$. An abundant expression of HSF1 protein has been observed in the tumor tissues of human prostate (19) and pancreas (22) or oral squamous cell carcinoma (OSCC) cells (24) compared to their normal counterparts. In addition, Khaleque et al (21) have shown that the protein level of HSF1 is upregulated in breast carcinoma sections and that HSF1 co-localizes and binds to the corepressor metastasisassociated 1 (MTA1) protein in this cancer.

In 2007, Dai et al (27) found that HSF1, a non-oncogene, plays a major role in enabling the initiation and maintenance of cancer in several mouse tumor models. Knockdown of HSF1 using short hairpin RNA (shRNA) technology effectively inhibited cell viability in several cancer cell lines, whereas it had no effect on normal human fibroblasts. They concluded that HSF1 function helps to maintain the growth and survival of human cancer cells (27). Similar results were reported when other in vitro experiments were performed using melanoma cells $(29,36)$, OSCC cells (37), pancreatic cancer cells (22) and human epidermal growth factor receptor 2 (HER2)positive cancer cells (58) (Table II). Although the molecular mechanisms by which HSF1 regulates cellular proliferation and survival in cancer cells are less understood, genome-wide transcriptome studies have indicated that HSF1 regulates numerous other targets in addition to HSPs $(25,59-62)$.

Basic experiments using HSF1 KO mice clearly demonstrate that this protein participates in the development of skin tumors (27) and hepatocellular carcinomas (28) induced by 7,12-dimethylbenzanthracene (DMBA) and diethylnitrosamine 
Table I. Examples of nonclinical and clinical studies elevating HSF1.

\begin{tabular}{|c|c|c|}
\hline Cancer type & Observations & Author/(Ref.) \\
\hline \multicolumn{3}{|l|}{ Nonclinical studies } \\
\hline Prostate cancer specimens & Protein level is upregulated in the malignant prostate epithelial cells & Hoang et al (19) \\
\hline Prostate carcinoma cells & Protein level is elevated in the more highly malignant carcinoma cells & $\begin{array}{l}\text { Hoang et al (19) } \\
\text { Tang et al (20) }\end{array}$ \\
\hline Breast carcinoma sections & Protein level is upregulated and it co-localizes with MTA1 protein & Khaleque et al (21) \\
\hline Pancreatic cancer tissues & Protein level is upregulated in cancer tissues & Dudeja et al (22) \\
\hline OSCC cells & Protein and mRNA levels are elevated in carcinoma cells & Ishiwata et al (24) \\
\hline \multicolumn{3}{|l|}{ Clinical studies } \\
\hline Breast cancer & Nuclear level is associated with poor prognosis & Santagata et al (23) \\
\hline OSCC & Nuclear level is related to tumor size & Ishiwata et al (24) \\
\hline Breast, colon or lung cancer & $\begin{array}{l}\text { Active HSF1 transcriptional program is strongly associated } \\
\text { with metastasis and mortality }\end{array}$ & Mendillo et al (25) \\
\hline Breast cancer & mRNA level is correlated with grade, metastasis and poor prognosis & Gabai et al (26) \\
\hline
\end{tabular}

HSF1, heat shock transcription factor 1; OSCC, oral squamous cell carcinoma; MTA1, metastasis-associated 1.

Table II. Examples of experimental anticancer effects targeting HSF1.

\begin{tabular}{|c|c|c|c|}
\hline Cancer type & Treatment & Effect & Author/(Ref.) \\
\hline \multicolumn{4}{|l|}{ HSF1 silencing } \\
\hline Pancreatic cancer cells & Quercetin & Quercetin increases the apoptosis of cells & Aghdassi et al (64) \\
\hline Pancreatic cancer xenografts & Quercetin & Quercetin reduces the growth of tumors & Aghdassi et al (64) \\
\hline Colon cancer xenografts & KRIBB11 & KRIBB11 inhibits the growth of tumors & Yoon et al (65) \\
\hline Pancreatic cancer xenografts & Ly 101-4B & Ly101-4B inhibits the growth of tumors & Xia et al (66) \\
\hline $\begin{array}{l}\text { Mammary epithelial cell } \\
\text { xenografts }\end{array}$ & shRNA & $\begin{array}{l}\text { shRNA inhibits HER2-induced cellular } \\
\text { transformation and tumorigenesis }\end{array}$ & Meng et al (59) \\
\hline Melanoma cell xenografts & shRNA & shRNA inhibits the growth of melanomas & Fujimoto et al (29) \\
\hline Cancer cells & shRNA & shRNA inhibits the growth of cancer cells & Dai et al (27) \\
\hline HER2-positive cancer cells & shRNA & shRNA decreases proliferation of cancer cells & Meng et al (59) \\
\hline Melanoma cells & shRNA & shRNA inhibits the growth of melanoma cells & $\begin{array}{l}\text { Nakamura et al (36) } \\
\text { Fujimoto et al (29) }\end{array}$ \\
\hline Pancreatic cancer cells & siRNA & siRNA decreases the viability of cancer cells & Dudeja et al (22) \\
\hline OSCC cells & siRNA & siRNA inhibits the growth of OSCC cells & Tabuchi et al (37) \\
\hline \multicolumn{4}{|l|}{ Genetically modified mice } \\
\hline $\begin{array}{l}\text { Chemical-induced skin } \\
\text { carcinomas }\end{array}$ & HSF1 KO mouse & KO inhibits carcinogen-induced tumorigenesis & Dai et al (27) \\
\hline $\begin{array}{l}\text { p53-deficient spontaneous } \\
\text { tumors }\end{array}$ & $\begin{array}{l}\text { HSF1 KO mouse } x \\
\text { p53 }^{\text {R172H }} \text { mouse }^{\mathrm{a}}\end{array}$ & $\begin{array}{l}\text { KO increases survival and alters the } \\
\text { distribution of tumor types }\end{array}$ & Dai et al (27) \\
\hline $\begin{array}{l}\text { p53-deficient spontaneous } \\
\text { tumors }\end{array}$ & $\begin{array}{l}\text { HSF1 KO mouse } \mathrm{x} \\
\text { p53 KO mouse }\end{array}$ & KO selectively decreases lymphomas & Min et al (63) \\
\hline $\begin{array}{l}\text { Chemical-induced } \\
\text { hepatocellular carcinomas }\end{array}$ & HSF1 KO mouse & KO inhibits carcinogen-induced tumorigenesis & Jin et al (28) \\
\hline NeuT-induced breast tumors & $\begin{array}{l}\text { HSF1 KO mouse } x \\
\text { Her2/NeuT mouse }^{c}\end{array}$ & KO suppresses tumor progression & Gabai et al (26) \\
\hline
\end{tabular}

${ }^{\mathrm{a}} \mathrm{HSF} 1 \mathrm{KO}$ mice were crossed with knockin mice caring the $\mathrm{p} 53^{\mathrm{R} 172 \mathrm{H}}$ hot spot mutation. ${ }^{\mathrm{b}} \mathrm{HSF} 1 \mathrm{KO}$ mice were crossed with $\mathrm{p} 53 \mathrm{KO}$ mice. ${ }^{c} \mathrm{HSF} 1 \mathrm{KO}$ mice were crossed with transgenic mice expressing HER2/NeuT (a rodent homolog of HER2 caring an activating mutation). HSF1, heat shock transcription factor 1; OSCC, oral squamous cell carcinoma; shRNA, short hairpin RNA; HER2, human epidermal growth factor receptor 2 ; $\mathrm{KO}$, knockout. 
(DEN), respectively. In line with these results, different animal experiments with genetically modified mice showed an essential role of HSF1 in tumor development $(26,27,63)$ (Table II).

Interest in the role of HSF1 in cancer has grown gradually over the last decade, and notable papers in clinical research have recently been published (23-26) (Table I). Santagata et al (23) indicated that nuclear localization and increased levels of HSF1 are well associated with poor prognosis in estrogen receptor (ER)-positive breast carcinomas. Ishiwata et al (24) reported that higher nuclear HSF1 expression was closely related to tumor size and histopathologic types in OSCCs. Moreover, high expression of HSF1 mRNA in human breast cancer was correlated with grade, metastasis, and poor prognosis, suggesting that HSF1 is involved in tumor progression (26). Mendillo et al (25) identified an HSF1-regulated transcriptional program specific to highly malignant cells and distinct from heat shock by using chromatin immunoprecipitation coupled with massively parallel DNA sequencing. They established an 'HSF1-cancer signature' of 456 genes that were bound by HSF1 near their transcription start sites, and found that this HSF1 cancer program is active in several types of tumors and is strongly associated with metastasis and mortality (25) (Table I).

To date, several types of HSF1 inhibitors have been developed, and their inhibitory effects to cancer have been reported (35,64-68) (Table II). Quercetin, a plant-derived flavonoid, is shown to increase apoptosis and to reduce the growth of pancreatic cancer cells (64). Meanwhile, this flavonoid inhibits multiple target proteins, such as HSF1, Hsp70 and NF- $\mathrm{BB}$ (67-69). In a colon cancer xenograft mouse model, a newly synthesized KRIBB11 $\left[N^{2}-\left(1 H\right.\right.$-indazole-5-yl)- $N^{6}$ methyl-3-nitropyridine-2,6-diamine] significantly inhibited the growth of colon cancer, and its mechanism is considered to inhibit Hsp70 synthesis through suppression of HSF1 function by impairing the recruitment of positive transcription elongation factor $\mathrm{b}$ (p-TEFb) to the Hsp70 promoter (65). Ly101-4B, a novel triazole nucleoside analog, targets the heat shock response pathway by downregulation of HSF1 and consequential inhibition of HSPs, Hsp27, Hsp70 and Hsp90 (66). This compound caused the shutdown of several oncogenic pathways and caspase-dependent apoptosis, resulting in a potent anticancer effect in pancreatic cancer cell xenografts $(66,68)$. Similar results were obtained when shRNA for HSF1 was used $(29,59)$. These findings have helped to elucidate the specific role of HSF1 in the pathogenesis of cancer.

\section{HSF1 and hyperthermia}

HT has been considered a possible mode of cancer treatment (1). HT in combination with radiotherapy and/or chemotherapy has been used for various types of cancer, and the anticancer effects of such combinations have been verified in many clinical trials (1-7). One of the problems with HT therapy is the acquisition of thermoresistance against heat stress (8-12). As the expression of HSPs is primarily mediated at the transcription level by HSF1 $(17,18)$, it is predicted that downregulation of HSPs by the targeting of HSF1 will render tumors more sensitive to HT. Examples of the enhancement of HT sensitivity targeting HSF1 are summarized in Table III. It is well established that preconditioning of the cells with mild-HT results in an increase in HSP levels and 
leads to the acquisition of thermoresistance to HT. However, McMillan et al (30) were the first show that targeted disruption of HSF1 abolished thermoresistance against lethal HT at $45^{\circ} \mathrm{C}$ for $40 \mathrm{~min}$ in preheated $\left(43^{\circ} \mathrm{C}, 30 \mathrm{~min}\right)$ mouse embryonic fibroblast (MEF) cells from HSF1-KO mice. In the MEF cells, silencing HSF1 prevented induction of the expression of two inducible HSPs, Hsp27 and Hsp70, whereas the expression of the constitutive HSPs, Hsp60 and Hsc70, remained constant, suggesting that inducible HSPs are involved in acquired cellular resistance to HT (30). Consistent with this result, functional silencing by using $\mathrm{KO}$ mice $(31,32)$ or a dominantnegative (DN) mutant (33) prevented the thermoresistance to $\mathrm{HT}$ in MEF and bone marrow progenitor (BMP) cells or breast cancer cells, respectively (Table III).

Of note, targeting of HSF1 has been suggested to enhance the sensitivity to HT under basic experimental conditions (29-38). We recently showed that silencing HSF1 using small interfering RNA (siRNA) enhances the mild HT $\left(42^{\circ} \mathrm{C}\right.$, $90 \mathrm{~min})$ and $\mathrm{HT}\left(44^{\circ} \mathrm{C}, 90 \mathrm{~min}\right)$ sensitivity in human OSCC cells (37). Significant enhancement of the HT sensitivity has also been observed in HSF1-silenced cervical cancer (35) and melanoma cells $(29,36)$ treated with triptolide, an inhibitor of the human heat shock response, and shRNA for HSF1, respectively. In addition, although HT had only a slight effect on tumor growth in breast cancer cell xenografts, functional defect of HSF1 by its DN mutant in combination with HT had a lower tumorigenic capacity than the control tumors (33).

The effects of inhibition of HSH1 on the hyperthermochemotherapy have been reported $(36,38)$. When HSF1 of cervical cancer cells was silenced by siRNA technology, the silencing in combination with hyperthermochemotherapy (treatment at $43^{\circ} \mathrm{C}$ for 60 min plus cisplatin) markedly increased the level of apoptosis compared to hyperthermochemotherapy alone (38). In human melanoma cells, although HSF1 silencing did not sensitize to chemotherapy with dacarbazine, it significantly enhanced the sensitivity to this chemotherapy in combination with HT $\left(42^{\circ} \mathrm{C}, 60 \mathrm{~min}\right)(36)$. These results strongly suggest that silencing HSF1 can enhance the sensitivity to either HT or hyperthermochemotherapy. It is also noteworthy that combined treatment with HT and cisplatin effectively suppresses the activation of HSF1 in human glioblastoma cells (70).

\section{Discussion}

Although HT is considered to be a promising approach in cancer therapy, the thermoresistance due to the increase in HSPs in cancer cells remains a disadvantage, reducing the effects of HT in clinical treatment. The expression of HSPs is mainly regulated by HSF1 $(17,18)$. HSPs $(12,56,57)$ as well as HSF1 (19-26) are abundantly expressed in human cancer cells of various origins, and HSPs $(12,56,57)$ and HSF1 (12,25-29) play critical roles in the initiation, proliferation and maintenance of cancer. Thus, the combination of HT and HSF1-targeting may be an attractive option and worthy of further investigation. As expected, the inhibition of functions of HSF1 by using gene targeting or HSF1 inhibitors was shown to reduce the acquisition of thermoresistance (30-33) and to sensitize tumors to HT-induced cell death (29-38). In the near future, targeting HSF1 $(67,68)$ in combination with HT may come to be a promising approach for the treatment of cancer.

\section{References}

1. Hall EJ (ed): Hyperthermia. In: Radiobiology for the Radiologist. 5th edition. Lippincott Williams and Wilkins, Philadelphia, PA, pp495-520, 2000.

2. van der Zee J, González González D, van Rhoon GC, van Dijk JD, van Putten WL and Hart AA: Comparison of radiotherapy alone with radiotherapy plus hyperthermia in locally advanced pelvic tumours: a prospective, randomised, multicentre trial. Dutch Deep Hyperthermia Group. Lancet 355: 1119-1125, 2000.

3. Harima Y, Nagata K, Harima K, Ostapenko VV, Tanaka Y and Sawada S: A randomized clinical trial of radiation therapy versus thermoradiotherapy in stage IIIB cervical carcinoma. Int J Hyperthermia 17: 97-105, 2001.

4. Wust P, Hildebrandt B, Sreenivasa G, et al: Hyperthermia in combined treatment of cancer. Lancet Oncol 3: 487-497, 2002.

5. Issels RD: Hyperthermia adds to chemotherapy. Eur J Cancer 44 2546-2554, 2008.

6. Zagar TM, Oleson JR, Vujaskovic Z, et al: Hyperthermia combined with radiation therapy for superficial breast cancer and chest wall recurrence: a review of the randomised data. Int J Hyperthermia 26: 612-617, 2010.

7. Westermann A, Mella O, Van Der Zee J, et al: Long-term survival data of triple modality treatment of stage IIB-III-IVA cervical cancer with the combination of radiotherapy, chemotherapy and hyperthermia - an update. Int J Hyperthermia 28: 549-553, 2012.

8. Rhee JG, Schuman VL, Song CW and Levitt SH: Difference in the thermotolerance of mouse mammary carcinoma cells in vivo and in vitro. Cancer Res 47: 2571-2575, 1987.

9. Dings RP, Loren ML, Zhang Y, et al: Tumour thermotolerance, a physiological phenomenon involving vessel normalisation. Int J Hyperthermia 27: 42-52, 2011.

10. Li GC, Mivechi NF and Weitzel G: Heat shock proteins, thermotolerance, and their relevance to clinical hyperthermia. Int $\mathrm{J}$ Hyperthermia 11: 459-488, 1995.

11. Cheng L, Smith DJ, Anderson RL and Nagley P: Human neuroblastoma SH-SY5Y cells show increased resistance to hyperthermic stress after differentiation, associated with elevated levels of Hsp72. Int J Hyperthermia 27: 415-426, 2011.

12. Mosser DD and Morimoto RI: Molecular chaperones and the stress of oncogenesis. Oncogene 23: 2907-2918, 2004.

13. Lindquist $\mathrm{S}$ and Craig EA: The heat-shock proteins. Annu Rev Genet 22: 631-677, 1988.

14. Hartl FU: Molecular chaperones in cellular protein folding. Nature 381: 571-579, 1996.

15. Beere HM: 'The stress of dying': the role of heat shock proteins in the regulation of apoptosis. J Cell Sci 117: 2641-2651, 2004.

16. Richter K, Haslbeck M and Buchner J: The heat shock response: life on the verge of death. Mol Cell 40: 253-266, 2010.

17. Morimoto RI: Regulation of the heat shock transcriptional response: cross talk between a family of heat shock factors, molecular chaperones, and negative regulators. Genes Dev 12: 3788-3796, 1998.

18. Akerfelt M Morimoto RI and Sistonen L: Heat shock factors: integrators of cell stress, development and lifespan. Nat Rev Mol Cell Biol 11: 545-555, 2010.

19. Hoang AT, Huang J, Rudra-Ganguly N, et al: A novel association between the human heat shock transcription factor 1 (HSF1) and prostate adenocarcinoma. Am J Pathol 156: 857-864, 2000.

20. Tang D, Khaleque MA, Jones EL, et al: Expression of heat shock proteins and heat shock protein messenger ribonucleic acid in human prostate carcinoma in vitro and in tumors in vivo. Cell Stress Chaperones 10: 46-58, 2005.

21. Khaleque MA, Bharti A, Gong J, et al: Heat shock factor 1 represses estrogen-dependent transcription through association with MTA1. Oncogene 27: 1886-1893, 2008.

22. Dudeja V, Chugh RK, Sangwan V, et al: Prosurvival role of heat shock factor 1 in the pathogenesis of pancreatobiliary tumors. Am J Physiol Gastrointest Liver Physiol 300: G948-G955, 2011.

23. Santagata S, Hu R, Lin NU, et al: High levels of nuclear heatshock factor 1 (HSF1) are associated with poor prognosis in breast cancer. Proc Natl Acad Sci USA 108: 18378-18383, 2011.

24. Ishiwata J, Kasamatsu A, Sakuma K, et al: State of heat shock factor 1 expression as a putative diagnostic marker for oral squamous cell carcinoma. Int J Oncol 40: 47-52, 2012.

25. Mendillo ML, Santagata S, Koeva M, et al: HSF1 drives a transcriptional program distinct from heat shock to support highly malignant human cancers. Cell 150: 549-562, 2012. 
26. Gabai VL, Meng L, Kim G, Mills TA, Benjamin IJ and Sherman MY: Heat shock transcription factor Hsf1 is involved in tumor progression via regulation of hypoxia-inducible factor 1 and RNA-binding protein HuR. Mol Cell Biol 32: 929-940, 2012.

27. Dai $C$, Whitesell L, Rogers AB and Lindquist S: Heat shock factor 1 is a powerful multifaceted modifier of carcinogenesis. Cell 130: 1005-1018, 2007.

28. Jin X, Moskophidis D and Mivechi NF: Heat shock transcription factor 1 is a key determinant of HCC development by regulating hepatic steatosis and metabolic syndrome. Cell Metab 14: 91-103, 2011.

29. Fujimoto M, Takaki E, Takii R, et al: RPA Assists HSF1 access to nucleosomal DNA by recruiting histone chaperone FACT. Mol Cell 48: 182-194, 2012.

30. McMillan DR, Xiao X, Shao L, Graves K and Benjamin IJ: Targeted disruption of heat shock transcription factor 1 abolishes thermotolerance and protection against heat-inducible apoptosis J Biol Chem 273: 7523-7528, 1998.

31. Luft JC, Benjamin IJ, Mestril R and Dix DJ: Heat shock factor 1-mediated thermotolerance prevents cell death and results in G2/M cell cycle arrest. Cell Stress Chaperones 6: 326-336, 2001

32. Zhang Y, Huang L, Zhang J, Moskophidis D and Mivechi NF: Targeted disruption of hsfl leads to lack of thermotolerance and defines tissue-specific regulation for stress-inducible Hsp molecular chaperones. J Cell Biochem 86: 376-393, 2002.

33. Wang JH, Yao MZ, Gu JF, Sun LY, Shen YF and Liu XY: Blocking HSF1 by dominant-negative mutant to sensitize tumor cells to hyperthermia. Biochem Biophys Res Commun 290: 1454-1461, 2002

34. Xia W, Vilaboa N, Martin JL, Mestril R, Guo Y and Voellmy R: Modulation of tolerance by mutant heat shock transcription factors. Cell Stress Chaperones 4: 8-18, 1999.

35. Westerheide SD, Kawahara TL, Orton K and Morimoto RI: Triptolide, an inhibitor of the human heat shock response that enhances stress-induced cell death. J Biol Chem 281: 9616-9622, 2006.

36. Nakamura Y, Fujimoto M, Hayashida N, Takii R, Nakai A and Muto M: Silencing HSF1 by short hairpin RNA decreases cell proliferation and enhances sensitivity to hyperthermia in human melanoma cell lines. J Dermatol Sci 60: 187-192, 2010.

37. Tabuchi Y, Furusawa Y, Wada S, Ohtsuka K and Kondo T: Silencing heat shock transcription factor 1 using small interfering RNA enhances mild hyperthermia and hyperthermia sensitivity in human oral squamous cell carcinoma cells. Thermal Med 27: 99-108, 2011

38. Rossi A, Ciafrè S, Balsamo M, Pierimarchi $\mathrm{P}$ and Santoro MG Targeting the heat shock factor 1 by RNA interference: a potent tool to enhance hyperthermochemotherapy efficacy in cervical cancer. Cancer Res 66: 7678-7685, 2006.

39. Sakurai $\mathrm{H}$ and Enoki Y: Novel aspects of heat shock factors: DNA recognition, chromatin modulation and gene expression. FEBS J 277: 4140-4149, 2010.

40. Westwood JT, Clos J and Wu C: Stress-induced oligomerization and chromosomal relocalization of heat-shock factor. Nature 353: 822-827, 1991.

41. Mariner PD, Walters RD, Espinoza CA, et al: Human Alu RNA is a modular transacting repressor of mRNA transcription during heat shock. Mol Cell 29: 499-509, 2008.

42. Spriggs KA, Bushell M and Willis AE: Translational regulation of gene expression during conditions of cell stress. Mol Cell 40: 228-237, 2010

43. Furusawa Y, Tabuchi Y, Wada S, Takasaki I, Ohtsuka K and Kondo T: Identification of biological functions and gene networks regulated by heat stress in U937 human lymphoma cells. Int J Mol Med 28: 143-151, 2011.

44. Tabuchi Y, Wada S, Furusawa Y, Ohtsuka K and Kondo T: Gene networks related to the cell death elicited by hyperthermia in human oral squamous cell carcinoma HSC-3 cells. Int J Mol Med 29: 380-386, 2012.

45. Tabuchi Y, Furusawa Y, Kariya A, Wada S, Ohtsuka K and Kondo T: Common gene expression patterns responsive to mild temperature hyperthermia in normal human fibroblastic cells. Int J Hyperthermia 29: 38-50, 2013.

46. Jin X, Eroglu B, Moskophidis D and Mivechi NF: Targeted deletion of Hsf1, 2, and 4 genes in mice. Methods Mol Biol 787: $1-20,2011$.
47. Xiao X, Zuo X, Davis AA, et al: HSF1 is required for extraembryonic development, postnatal growth and protection during inflammatory responses in mice. EMBO J 18: 5943-5952, 1999.

48. Homma S, Jin X, Wang G, et al: Demyelination, astrogliosis, and accumulation of ubiquitinated proteins, hallmarks of CNS disease in hsf1-deficient mice. J Neurosci 27: 7974-7986, 2007.

49. Zou Y, Zhu W, Sakamoto M, et al: Heat shock transcription factor 1 protects cardiomyocytes from ischemia/reperfusion injury. Circulation 108: 3024-3030, 2003

50. Takaki E, Fujimoto M, Sugahara K, et al: Maintenance of olfactory neurogenesis requires HSF1, a major heat shock transcription factor in mice. J Biol Chem 281: 4931-4937, 2006.

51. Kallio M, Chang Y, Manuel M, et al: Brain abnormalities, defective meiotic chromosome synapsis and female subfertility in HSF2 null mice. EMBO J 21: 2591-2601, 2002.

52. Fujimoto M, Izu H, Seki K, et al: HSF4 is required for normal cell growth and differentiation during mouse lens development. EMBO J 23: 4297-4306, 2004.

53. Bishop NA and Guarente L: Genetic links between diet and lifespan: Shared mechanisms from yeast to humans. Nat Rev Genet 8: 835-844, 2007.

54. Neef DW, Jaeger AM and Thiele DJ: Heat shock transcription factor 1 as a therapeutic target in neurodegenerative diseases. Nat Rev Drug Discov 10: 930-944, 2011

55. Hanahan D and Weinberg RA: Hallmarks of cancer: the next generation. Cell 144: 646-674, 2011.

56. Ciocca DR and Calderwood SK: Heat shock proteins in cancer: diagnostic, prognostic, predictive, and treatment implications. Cell Stress Chaperones 10: 86-103, 2005.

57. Ciocca DR, Arrigo AP and Calderwood SK: Heat shock proteins and heat shock factor 1 in carcinogenesis and tumor development: an update. Arch Toxicol 87: 19-48, 2013.

58. Tabuchi Y, Kariya A, Yunoki T and Kondo T: Genes involved in the cell death induced by knockdown of heat shock transcription factor 1 in human oral squamous cell carcinoma HSC-3 cells. Thermal Med 28: 29-42, 2012.

59. Meng L, Gabai VL and Sherman MY: Heat-shock transcription factor HSF1 has a critical role in human epidermal growth factor receptor-2-induced cellular transformation and tumorigenesis. Oncogene 29: 5204-5213, 2010.

60. Hahn JS, Hu Z, Thiele DJ and Iyer VR: Genome-wide analysis of the biology of stress responses through heat shock transcription factor. Mol Cell Biol 24: 5249-5256, 2004.

61. Trinklein ND, Murray JI, Hartman SJ, Botstein D and Myers RM: The role of heat shock transcription factor 1 in the genome-wide regulation of the mammalian heat shock response. Mol Biol Cell 15: $1254-1261,2004$

62. Page TJ, Sikder D, Yang L, et al: Genome-wide analysis of human HSF1 signaling reveals a transcriptional program linked to cellular adaptation and survival. Mol Biosyst 2: 627-639, 2006

63. Min JN, Huang L, Zimonjic DB, Moskophidis D and Mivechi NF: Selective suppression of lymphomas by functional loss of Hsf 1 in a p53-deficient mouse model for spontaneous tumors. Oncogene 26: 5086-5097, 2007.

64. Aghdassi A, Phillips P, Dudeja V, et al: Heat shock protein 70 increases tumorigenicity and inhibits apoptosis in pancreatic adenocarcinoma. Cancer Res 67: 616-625, 2007.

65. Yoon YJ, Kim JA, Shin KD, et al: KRIBB11 inhibits HSP70 synthesis through inhibition of heat shock factor 1 function by impairing the recruitment of positive transcription elongation factor b to the hsp70 promoter. J Biol Chem 286: 1737-1747, 2011.

66. Xia Y, Liu Y, Rocchi P, et al: Targeting heat shock factor 1 with a triazole nucleoside analog to elicit potent anticancer activity on drug-resistant pancreatic cancer. Cancer Lett 318: 145-153, 2012.

67. Whitesell L and Lindquist S: Inhibiting the transcription factor HSF1 as an anticancer strategy. Expert Opin Ther Targets 13: 469-478, 2009

68. Xia Y, Rocchi P, Iovanna JL and Peng L: Targeting heat shock response pathways to treat pancreatic cancer. Drug Discov Today 17: 35-43, 2012.

69. Chen SS, Michael A and Butler-Manuel SA: Advances in the treatment of ovarian cancer: a potential role of antiinflammatory phytochemicals. Discov Med 13: 7-17, 2012.

70. Matsumoto H, Hayashi S, Shioura H, et al: Suppression of heatinduced HSF activation by CDDP in human glioblastoma cells. Int J Radiat Oncol Biol Phys 41: 915-920, 1998. 